II.

Aus dem Kgl. medicinisch-poliklinischen Institut der Universität Berlin (Director: Geh. Med.-Rath Prof. Dr. Goldscheider).

\title{
Die Giftigkeit des Methyl- und Aethylalkohols.
}

Von

Prof. Dr. Alexander Langgaard.

Bekanntlich steigert sich die Wirkung bei den einatomigen Alkoholen mit der Zunahme der Kohlenstoffatome, d. h. der Propylalkohol wirkt stärker als der Aethylalkohol, der Butylalkohol stärker als der Propylalkohol, der Amylalkohol stärker als der Butylalkohol. Fine Ausnahme soll allein der Methylalkohol machen, welcher giftiger sein soll als der Aethylalkohol. Wie unzureichend aber unsere Kenntnisse über die Giftigkeit des Methylalkohols waren, haben die Verhandlungen im Process Scharmach gezeigt.

Ich habe seit Januar eine grössere Anzahl ausgedehnter vergleichender Versuche über die Giftigkeit des Methyl- und Aethylalkohols gemacht, deren Resultate ich kurz in No. 36 der Berliner klin. Wochenschrift mitgetheilt habe. Nun will ich in Folgendem über die Ergebnisse meiner Untersuchungen ausführlich berichten.

Ich habe ausschliesslich Kaninchen benutzt und zunächst mit kleinen Dosen ( $3 \mathrm{ccm}$ pro Kilo Thier) begonnen, die täglich bis zum Eintritt des Todes gegeben wurden. Ich habe dann die Dosen gesteigert auf 5, 6 und $8 \mathrm{ccm}$ pro Kilo und habe schliesslich, um wirklich die einmalige letale Dosis festzustellen, Dosen von 10, 12 und 14 ecm gegeben.

Als Methylalkohol kam der käufliche acetonfreie zur Anwendung, der, mit der gleichen Henge Wasser verdünnt, den 'Thieren in den Hagen gegeben wurde. Ebenso wurde natürlich auch der Aethylalkohol gegeben.

In Folgendem gebe ich die Versuchsprotokolle wieder. Ich will hier gleich vorweg bemerken, dass ich die Protokolle der Versuche mit den grossen Dosen 10 und $12 \mathrm{ccm}$ nicht in ihrem ganzen Umfange wiedergebe. Erstens, weil sie zu lang sind, sie erstrecken sich: Versuch 1 vom 2. 7. bis 27. 7., Versuch 2 vom 5. 7. bis 27. 7., Versuch 3 vom 8. 7. bis 27. 7., Versuch 4 vom 10. 7. bis 27. 7., Versuch 5 vom 15. 7. bis 27. 7., und zweitens, weil in dem weiteren Verlauf der Versuche keine Veränderungen in dem Zustande der Thiere eintraten. 


\section{Methylalkohol acetonfrei, 3 r'm pro Kilo.}

15. 3. 1912. Kaninclien, $1900 \mathrm{~g}$.

11 h 15. Frhält $5,7 \mathrm{ccm}$.

11 h 40. Reflexerregbarkeit gesteigert. Unruhe.

$11 \mathrm{~h} 43$. Hüpft umher.

11 h 45 . Ijegt sich auf den Bauch.

$11 \mathrm{~h} 46$. Richtet sich wieder auf.

$11 \mathrm{~h} 50$. Sehr unruhig.

$11 \mathrm{~h} 55$. Ifat sich auf den Bauch grelert. Resp. 87. I'uls $\mathbf{2 4 0 .}$

12 h 00 . Richtot sich auf. Sehr erregt. Ohrgefüsse stark erweitert.

12 ly 30. Leegrt sich auf den Bauch. Sehr benommen. Resp. 64. Puls 240.
$12 \mathrm{~h} 32$. In den Sehwanz grekniffen, springt es sufort auf.

12 h 35 . Hüpft umher. Sehr erregt.

$12 \mathrm{~h} 38$. Will sich an der Wand aufrichten, fällt aber dabei um.

12 h 45 . Puls 250.

12 h 50 . Sitzt aufretht. Reflexerregbarkeit gesteigert.

$12 \mathrm{~h} 55$. 'Leicht benommen.

1 h 00 . Sitzt horkend, sehr benummen.

1 h 05 . Richtet sich auf und hüpft fort.

$1 \mathrm{~h}$ 10. Sehr unruhigr.

$1 \mathrm{~h} 15$. Wird in den Küfig gesetzt.

\section{3. $1912.1900 \mathrm{~g}$.}

$11 \mathrm{~h} 00$. Sehr erregt. Frhält $5,7 \mathrm{ccm}$.

$11 \mathrm{~h}$ 10. Hat sich auf den Bauch gelegt. Resp. 116. Kussmaul.

$11 \mathrm{~h} 15$. Hat sich aufgerichtet.

11 h 18 . Legt sich wieder nieder.

$11 \mathrm{~h} 30$. Hat sich aufgeriehtet.

1 I 32 . Puls 257.

$11 \mathrm{~h} 34$. Iegt sich auf den Bauch.

11 h 36 . Richtet sich wieder auf und hüpft.

$12 \mathrm{~h} 00$. Legt sich wieder auf den Bauch.
12 h 20 . Richtet sich plïtzlich auf und hiupft.

$12 \mathrm{~h} 25$. Sitzt, leicht benommen.

12 h 32 . Resp. 47.

12 h 40 . Hüpft unher.

$12 \mathrm{~h} 43$. Legt sich wieder auf den Banch.

1 h 20. Liegt auf dem Bauche. Resp. at.

1 h 28. kichtet sieh auf.

1 h 30. Sitzt. Vorderkïrper hoch aufgerichtet.

\section{3. $1912.1900 \mathrm{~s}$.}

11 h 05 . Sitzt, Vorderkörper aufgerichtet. Reflexerregbarkeit resteigert.

$11 \mathrm{~h}$ 15. Legt sich auf den Bauch. Resp. 66.

11 h 20. Ganz benommen. Fuls 260.

$11 \mathrm{~h} 40$. Frhält $5,7 \mathrm{ccm}$.

$11 \mathrm{~h} 50$. Legt sich auf den Bauch.

12 h 00 . Richtet sich auf.
12 h 10. Hat sich wieder gelergt. Sehr müile.

12 h 20 . Hat sich wieder aufrerichtet.

$12 \mathrm{~h} 30$. Hat sich wieder gelegt.

$12 \mathrm{~h} 40$. Hichtet sich wieder auf. Puls $\mathbf{2 6 0}$ Resp. 4.

12 h 45. Legt sich wieder auf den Bauch.

$1 \mathrm{~h}$ 1.5. Wird in den liafig gelegt.

\section{3. $1912.1800 \mathrm{~s}$.}

11 h 00 . Sitzt ruhicr.

$11 \mathrm{~h} 05$. Legt sich auf den Bauch.

11 h 10 . Richtet sich auf.

11 h 13. Puls 240. Resp. 64.

11 h 20 . Sitzt ruhig.

$11 \mathrm{~h} 30$. Erhält $5,4 \mathrm{ccm}$.

11 h 42. Liegt auf dem Bauche. Ohrgefïsse stark erweitert. Resp. 64, regelmässig tief.
12 h 20 . Liect auf dem Bauche. Benommen.

$12 \mathrm{~h} 22$. Resp. 50, regelmässir.

12. h 30 . Puls 260.

12 h 38 . Riclitet sich plötzlich auf.

12 h 55. Häptt umher.

1 h 00 . Fällt un, liegrt auf der seite.

1 15 . Richtet sich wieder auf.

\section{3. $1912.1800 \mathrm{~g}$.}

11 h 10 . Sitzt ruhig. Ohrgefiisse stark orweitert.

11 h 12. Jegt sich plötzlich.

11 h 20. Puls 250. Resp. 74.

11 h 30 . Erhält $5,4 \mathrm{ccm}$.

$11 \mathrm{~h} 35$. Hat sich relert.

11 h 37 . Resp. 116.

$11 \mathrm{~h} 42$. Richtet sich auf.

$11 \mathrm{~h} 44$. Reflexerregbarkeit gesteigert. Resp. ó8.
12 h 10. Jachdem das Thier sich wiederholt gelert und wicder aufgerichtet hat, liegt es sehr muide auf dem Bauche.

12 h 25 . liesp. 43 .

12 h 30 . Richtet sich auf und hüpft umher.

12 $h$ 40. Hat sich wieder auf den Bauch rclegt.

12 h 55. Sitit aufrecht. P'uls 272.

1 h 10 . Legt sich auf den Bauch. Resp. J4.

$1 \mathrm{~h} 30$. Wird in den Käfig gelegrt. 


\section{3. $1912.1800 \mathrm{~g}$.}

11 h 05 . Sitzt ruhig. Ohrgefiasse stark erweitert.

11 h 10. Puls 240. Resp. 45.

11 h 25 . Erhält $5,4 \mathrm{ccm}$.

$11 \mathrm{~h} 30$. Hat sich auf den Bauch gelegt.

11 h 35. Reflexerregbarkeit erhöht.

12 h 00 . Resp. 32.

12 h 05 . Richtet sich plötzlich auf.
12 h 15 . Sitzt selir benommen.

12 h 30 . Hüpt umher. Ohrgefässe stark erweitert.

$12 \mathrm{~h} 40$. Sehr unruhig. Reflexerregbarkeit erhöht.

$1 \mathrm{~h} 00$. Hüpft fortwährend umher.

1 h 15 . Sitzt ruhig.

$1 \mathrm{~h} 30$. Wird in den Käfig resetzt.

\section{3. $1912.1800 \mathrm{~g}$.}

11 h 10 . Sitzt aufrecht.

11 h 20. Juegt sich auf den Bauch. Resp. 41. Puls 260.

$11 \mathrm{~h} 40$. Frhält $5,4 \mathrm{~cm}$.

$11 \mathrm{~h} 50$. Liegt auf dem Bauche, müde.

11 h 55. Puls 230. Resp. 45.

$12 \mathrm{~h} 00$. Hat sich aufgerichtet, legt sich aber gleich wieder nieder.

\section{3. $1912,1700 \mathrm{~g}$.}

$11 \mathrm{l} 15$. Sitzt aufrecht, Ohrgefässe erweitert. Resp. ǒ 8 .

11 h 20. Sehr unruhig, hïpft umber.

11 h 30 . Firhält 5,1 ccm.

$11 \mathrm{~h} \mathrm{40.} \mathrm{Hat} \mathrm{bis} \mathrm{jetzt} \mathrm{ruhigr} \mathrm{gesessen,} \mathrm{legt}$ sich auf den Bauch.

$11 \mathrm{~h} 45$. Resp. 41, rerrelmässig. Starke Peristaltik.

\section{3. $1912.1700 \mathrm{gr}$.}

12 h 30. Sitzt aufrecht. Ohrufuasse stark erweitert. I'uls 210. Resp. 44.

12 h 40 . Erhält 5, $1 \mathrm{ccm}$.

12 h 55. Sitzt hockend, sehr benommen.

\section{3. $1912.1700 \mathrm{~g}$.}

1) h 05 . Sitzt ruhigr, leicht benommen.

$11 \mathrm{~h} \mathrm{10}$. Ohrgefässe stark erweitert. Resp. 37.

11 h 25 . Frhält $5,1 \mathrm{ccm}$.

$11 \mathrm{~h} 30$. I egt sich auf den Bauch. Schr benommen.

11 h 35. Puls 246. Resp. 39.

11 h 40. Wat sich plötzlich aufgerichtet und hïpft davon.

$11 \mathrm{~h} 45$. I eert sich plötzlich wieder auf den lauch.

\section{3. 1912. $1800 \mathrm{~g}$.}

11 h 40. Puls 240. Resp. 60.

$11 \mathrm{~h}+5$. Firhält $5,4 \mathrm{ccm}$.

12 h 05. Teert sich plötzlich auf den Bauch.

12 h 35. Sitzt aufrecht. (Jhrgefässe stark erweitert.

12 h 37 . Hüpft umher.

12 h 55. Legt sich plötzlich auf den Baueh.

12 h 57. Puls 240. Resp. 46.

28. 3. $1912.1800 \mathrm{~g}$.

12 h 00 . Unrubio, hijpft umher.

12 h 20 . Frhält $5,4 \mathrm{ecm}$.

12 h 30 . Sit?t aufrecht.

12 h 35 . Legt sich auf den Baueh. Resp. 38.

12 h 40. Richtet sich plotzlieh auf und liupft fort.

12 h 45 . Ohrgefäse stark erweit. Puls 246.
12 h 00 . Hüpft munter umher.

12 h 15 . Sitzt hockend. Reflexerreg barkeit gesteigert.

12 h 22. Legrt sirh auf den Bauch.

12 h 24 . Richtet sich wieder auf. Puls 250.

12 h 35 . Hüpft umher.

12 h 40 . Sehr unruhig. Huipft fortwährend umiler.

$1 \mathrm{~h}$ 05. Isegt sich auf den Bauch.

$1 \mathrm{~h} 15$. Wird in den häfig gesetat.

$1 \mathrm{~h} 00$. Iat sich wieder aufrerichtet.

1 h 05 . Jegrt sich plitzlich wieder auf den Bauch.

1 h 17 . Resp. 20. Sehrbenommen. Reagrirt auf Tupfen der Spïrhare.

I h 20. Sitzt hockend, cranz benummen.

$1 \mathrm{~h} 30$. Wird in den Kafifir gresetzt.

1 l 00 . Sitzt zusammengelsauert, henommen. Resp. 40.

1 ho 0 . Rightet sich auf.

1 h 10 . Sitzt aufreeht. Starke liaubewegungen.

I h 1․ Irüptt umher. 
29. 3. $1912.1800 \mathrm{gr}$.

$11 \mathrm{~h} 00$. Sitzt ruhig. Resp. 47.

11 h 48 . Erhält $5,4 \mathrm{ccm}$.

12 h 00 . Sitat aufrecht. Ohrgefässe stark erweitert.

11 h 45 . Erhält 5,1 cem.

12 h 00 . Sitzt benommen.

12 h 25 . Hüpft umher.
12 h 15. Hat sich auf den Bauch gelegt. liaubewegungen. Resp. 44.

12 h 20 . Liert ganz benommen auf dem Bauche.

30. 3. $1912.1700 \mathrm{~g}$.

12 h 30 . Sebr umruhig.

12 h 35. Sitzt ruhig. Resp. 36.

12 h 43. Sitzt ruhig, leicht benommen.

31. 3. $1912.1700 \mathrm{ir}$.

11 h 20. Sitzt aufrecht. Resp. ó6.

11 b 30 . Erhält $5,1 \mathrm{~cm}$.

$12 \mathrm{~h} 45$. Legt sich auf den Bauch.

12 h 50 . Versucht sich aufzurichten. Vorderpfoten gleiten aus.

11 h 40. Legt sich anf den Bauch.

11 h 45 . Richtet sich wieder auf.

11 h 48. Liegt auf dem Bauche, sehr benornmen.

1 hoo. lichtet sich auf.

1 h 05 . Lest sieh wieder auf den Bauch.

$1 \mathrm{~h} 30$. Wird in den häfirs gelegt.

12 h 30 . Richtet sich auf und hüpft umher.

\section{4. $1912.1400 \mathrm{~g}$.}

11 h 05 . Sitzi ruhig.

11 h 30. Sehr unruhigr. Hüpft umber.

11 h 35 . Erhält $4.2 \mathrm{~cm}$.

$11 \mathrm{~h} 45$. Liegt auf dem Bauche. Resp. 44.

$11 \mathrm{~h} \mathrm{55.} \mathrm{Sehr} \mathrm{unruhig,} \mathrm{hüpft} \mathrm{umher.}$

$12 \mathrm{~h} 00$. Legt sich auf den Bauch.

$12 \mathrm{~h}$ 10. Richtet sich plïtzlich auf und hüpt umher bis

1 h 00 . I egt sich auf den Bauch.

$1 \mathrm{~h} 12$. Richtet sich wieder auf und hüpft davon.

$1 \mathrm{~h} 20$. Jiegt benommen auf dem Bauche. Resp. 45.

1 h 30 . Wird in den häfig gelegt.

\section{4. $1912.1400 \mathrm{~g}$.}

$11 \mathrm{~h} 25$. Sitzt ruhig. Resp. 43.

11 h 50. Hat sich auf den Bauch gelegt.

11 h 35 . Erhält 4,2 ccm.

3. 4. $1912.1400 \mathrm{~g}$.

11 h 30 . Sehr matt.

$11 \mathrm{~h}$ 35. Frhält $4,2 \mathrm{~cm}$.

4. 4. 1912 . $1400 \mathrm{~g}$.

12 h 40. Resp. 56. Liegrt auf dem Bauche. 1 h 15 . Richtet sich auf. Starke Peristaltik.

$12 \mathrm{~h} 45$. Richtet sich auf und hïplt davon.

$12 \mathrm{~h}$ 55. Erhält $4,2 \mathrm{ccm}$.

1 h 10 . Hat sich gelegt. Hintertheil seitlich.

I h 20 . Ient sich wieder.

1 h 30 . Richtet sich auf. Sehr waekelir.

$1 \mathrm{~h} 40$. Wird in den Käligr gesetzt.

5. 4. 1912. Todt im Käfig vorgefunden. 19 mal 3 cem.

\section{Aethylalkohol 3 com pro Kilo.}

12 h 20 . Erhält $4,5 \mathrm{ccm}$.

12 h 30 . Grosse Linruhe.

12 h 40. Sitzt aufrecht.

1 h 00 . Nachdem es fortwährend umhergehuipft ist, setzt es sich plïtzlich hockend hiu und sucht Stütze an der Wand.

15. 4. $1912.1500 \mathrm{~g}$.

$11 \mathrm{~h} 50$. Sitzt ruhig.

$11 \mathrm{~h} 55$. Erhält $4,0 \mathrm{ccm}$.

12 h 00 . Resp. 30. Sehr unruhig. Müpft fortwährend

$$
\text { 16. 4. 1912. } 1500 \mathrm{~s} \text {. }
$$

11 h 50 . Erhält $4,5 \mathrm{ccm}$. 12 h 00 . Richtet sich auf.

$11 \mathrm{~h} 55$. Hat sich auf den Bauch gelegt.

1 h 05 . Richtet sich plitzlich an der Wand hoch auf, fält um, richtet sich aber sieich wieder auf.

1 h 12. Grosse Cincule, hüpft fortwährend umher.

1 h 25 . umher,

1 h 30 . Wird in den Käfir gesetat. 
$12 \mathrm{~h} 00$. Resp. 26 . Erhält $\mathbf{4 , 5} \mathrm{ccm}$.

\section{4. $1912.1500 \mathrm{~g}$.}

18. 4. $1912.1500 \mathrm{~g}$.

$1 \mathrm{~h} 10$. Sitzt ruhig.

1 h 15 . Erhält $4,5 \mathrm{ccm}$.

$1 \mathrm{~h}$ 30. Sitzt hockend, ganz benommen.

19. 4. $1912.1500 \mathrm{~g}$.

11 h 20. Sitzt hockend. Etwas erregt.

11 h 25. Resp. 95. Kussmaul.

11 h 40. Hüpft umher.

12 h 00 . Erhält $4,5 \mathrm{ccm}$.

$12 \mathrm{~h} 35$. Resp. 51. (ianz benommen.

12 h 40 . Hüpft umher.

11 h 25 . Sitat ruhir.

11 h 30. Erhält 4,3 ccm.

$11 \mathrm{~h} 35$. Resp. 62.

$12 \mathrm{~h} 00$. Lnruhig, hiipft umher.

$11 \mathrm{~h}$ 10. Jüpft umher.

11 h 25 . Frhält 43 cem.

11 h 50. Sitzt hockend.
20. 4. $1912.1450 \mathrm{~g}$.

12 h 30 . Sitzi benommen.

12 h 40 . Hüpft umher.

$12 \mathrm{~h} 50$. Benommen.

1 h 00 . In den Käfig gesetzt.

22. 4. 1912. $1450 \mathrm{gr}$.

12 h 15. Jaurt plötzlich über den Tisch.

12 h 25 . Sitzt hockend, ganz benommen.

23. 4. $1912.1450 \mathrm{~g}$.

11 h 40. Erhält 4,3 ecm.

12 h 40 . Hüpft bestïndig bis

12 h 10. Hat bis jetzt ruhigr gesessen. Jayt

12 h 20 . Hüpft umher. plötzlich über den Tisch. 1 h 65 . Wird in den Kälig gesetzt.

24. 4. $1912.1450 \mathrm{~g}$.

$11 \mathrm{~h} 00$. Sitzt ruhig.

12 h 40 . Sitzt hockend, ranz benommen.

$11 \mathrm{~h} 05$. Frhält $4,3 \mathrm{ccm}$.

12 h 45 . Richtet sich auf.

12 h 55 . Hürft umher.

1 h 00 . Sehr unruhig.

$1 \mathrm{~h}$ 10. Hüpft umher.

1 h 35 . Sitzt ruhig, ganz benommen. Resp. 41.

12 h 20 . Jagt plötzlich über den Tisch.

$12 \mathrm{~h} 30$. Hüpft umher. Kaubewegungen.

11 h 35 . Sitzt ruhig. Resp. 67.

25. 4. $1912.1400 \mathrm{~g}$.

$11 \mathrm{~h} 42$. Frhält $4.2 \mathrm{cem}$.

12 h 00 . Sitzt ruhig.

12 h 30. Sitzt hockend.

12 h 40 . Hüptt davon.

11 h 20. Sitzt ruhig.

$11 \mathrm{~h} 30$. Krrält $4,2 \mathrm{com}$.

11 h 35. Erhält 4,2 ccm.

$11 \mathrm{~h} 45$. Sitzt ruhig.

11 h 55 . Huipft umher.

12 h 05. Sitzt ranz benommen. Resp. 58.

12 h 10 . Cornealreflex erhalten.

11 h 30 . Frhiult $3,9 \mathrm{~cm}$.

12 h 00 . Sitzt ganz benommen.

$12 \mathrm{~h}$ 10. Cinruhire hijpft umber.

12 h 25. Solne unruhigg, hëpft umher.
12 h 50 . Sitat ruhig.

$1 \mathrm{~h} 00$. Hüpft umber.

1 h 0j. Sitzt hockend, benommen.

$1 \mathrm{~h} 20$. Hiipft umher.

1 h 30 . Wird in den Käfig gesetzt.

26. 4. 1912 . $1400 \mathrm{~g}$.

11 h 50 . Hiipft umher.

27. 4. 1912.1400 s.

12 h 30 . Sitzt hockend.

$12 \mathrm{~h} 40$. Hat sich aufrerichtet.

1 h 00 . Sitzt hockend, ganz benommen.

1 h 20 . Sitzt hockend, ganz benommen.

$1 \mathrm{~h} 30$. Wird in den Käfig gesetzt.

29. 4. $1912.1300 \mathrm{~g}$.

i h 10. Wihrend der ranzen 7eit umuhig umhergehïpft.

1 h 20 . Sitzt ruhig.

$1 \mathrm{~h} 30$. Wird in den Kitfig gesetzt. 
11 h 20 . Sitzt ruhig.

11 h 25. Resp. 62.

$11 \mathrm{~h} 38$. Frhält $3,9 \mathrm{ccm}$.

$11 \mathrm{~h} \mathrm{50.} \mathrm{Wird} \mathrm{unruhig,} \mathrm{hüpft} \mathrm{umher.}$

11 h 55. Jiegt sich anf den Bauch. Sehr benommen.

30. 4. $1912.1300 \mathrm{~g}$.

12 h 15 . Lnruhe.

12 h 30 . Hüpft umher

$1 \mathrm{~h} 20$. do. do.

$1 \mathrm{~h} 30$. Wird in den Käfig gesetzt.

1. 5. $1912.1300 \mathrm{~g}$.

II h 10. Sitzt ruhig, benommen. Resp. 59 .

11 h 25. Erhält 3,9 ccm.

$11 \mathrm{~h} 40$. Ganz benommen.

$12 \mathrm{~h} 45$. Sebr unruhig. Hüpft fortwährend umher.

$1 \mathrm{~h} 00$.' Sehr erregt.

1 h 15 . Sitzt zusammengrekauert, granz benommen.

2. 5. $1912.1300 \mathrm{~g}$.

11 h 15. Sitzt ruhig. Resp. 42.

$11 \mathrm{~h} 30$. Erhält $3,9 \mathrm{ccm}$.

$12 \mathrm{~h} 00$. Sitzt ganz benommen.

12 h 20 . Hüpft umber.

12 h 35. Sitzt ruhig, leicht benommen.

4. 5. $1912.1200 \mathrm{~g}$.

11 h 15. Sitzt ruhig, stützt den Kopf auf

12 h 20 . Hüpft davon.

12 h 25. Sitzt ruhig. Puls 240. Resp. 45.

1 h 00 . Ganz benommen. Reflexerregbarkeit gesteigert.

$11 \mathrm{~h}$ 25. Erhält $\mathbf{3 , 6} \mathrm{ccm}$.

11 h 30 . Sitzt zusammengekauert.

$11 \mathrm{~h} 40$. Hüpft davon.

12 h 00 . Sitzt ganz benommen.

1 h 20. Ganz benommen.

1 h 30 . Wird in den Käfig gesetzt.

5. 5. $1912.1200 \mathrm{~g}$.

12 h 30 . Sitzt zusammengekauert, ganz benommen.

12 h 37. Resp. 40.

12 h 50 . Erhält $3,6 \mathrm{ccm}$.

1 h 15. Liegrt auf dem Bauche. Ganz benommen.

$1 \mathrm{~h} 30$. In den Käfig gesetzt.

6. 5. $1912,1200 \mathrm{~g}$.

11 h 20. Sitztzusammengekauert. Resp. 35. | 11 h 30 . Frhält $3,6 \mathrm{ccm}$.

7. 5. $1912.1100 \mathrm{~g}$.

10. h 50. Leat sich auf den Bauch.

10 h 55. Resp. 40.

$11 \mathrm{~h} 10$. Erhält $3,3 \mathrm{ccm}$.

12 h 00 . Hat dic ganze Keit ruhig ge. sessen.

12 h 03 . IÏ̈pft davon.

\section{S. 5. $1912.1100 \mathrm{~g}$.}

$10 \mathrm{~h}$ 30. Sehr mait. Resp. 42.

11 h 00 . Grosse Lnruhe.

11 h 30 . Hüpft umher.

12 h 00 . Resp. 34.

10 h 50 . Grosse Unruhe.

9. 5. $1912.1000 \mathrm{~g}$.

12 h 00 . Sehr matt.

$12 \mathrm{~h} 25$. I'nruhe.

12 h 30. Sitzt ruhig. Resp. 38, dyspnoisch.

$12 \mathrm{~h} 40$. In den Käfg gesetzt.

12 h 15 . Erhält $3 \mathrm{ccm}$.

10. 5. 1912. Todt in Käfig vorgefunden. $23 \mathrm{mal} 3 \mathrm{ecm}$ pro Kilc.

\section{Methylalkohol. Vrsache der Vergiftung $3 \mathrm{ccm}$ pro Kilo.}

15. 3. 1912. Kaninchen, $1850 \mathrm{~g}$.

11 h 25 . Erhält 5,6 ccm.

$11 \mathrm{~h} \mathrm{40.} \mathrm{Ohrgefisse} \mathrm{stark} \mathrm{erweitert.} \mathrm{Legt}$ sich auf den Bauch.

11 h 45. Resp. 38. Kussmaul.

11 h 50. Liegt auf dem Bauche, sehr benommen. Reflexerregbar-

11 h 52. Puls 257.
$12 \mathrm{~h} 00$. Sitzt hockend, ganz benommen.

$12 \mathrm{~h} 20$. Hat sich aufgerichtet.

12 h 23. Legt sich auf den Bauch. Starke Peristaltik.

12 h 28. Puls 260, Resp. 40.

12 h 35 . Sitzt ganz benommen. Zutkt beim Kueifen in den Schwanz zusammen, bleibt aber ruhig sitzen. 


\section{3. 1912 (Fortsetzung).}

12 h 40. Sitzt ganz benommen. Resp. ganz 12 h 55. Hat sich auf den Bauch gelegt. flach, 45 .

12 h 42. Versucht zu hüpfen. Vorderpfoten gleiten aus.

12 h 49. Hat sich auf den Bauch gelegt. Puls 240.

12 h 52. Richtet sich wieder auf. Resp. 36 .

1 h 05 . Iiegt ganz benommen auf dem Bauche.

1 h 10. Hatsich auf gerichtet und hüpftfort.

1 h 12. Sitat hockend. Wird in den Kälig gesetzt.

16. 3. 1912. $1800 \mathrm{~g}$.

11 h 00 . Erhält $5,4 \mathrm{ccm}$.

11 h 10. Sitzt hockend.

$11 \mathrm{~h}$ 20. Legt sich auf den Bauch.

11 h 22. Hat sich aufgerichtet.

$11 \mathrm{~h}$ 24. Legt sich wieder auf den Bauch. Hintertheil seitlich.

11 h 25. Rep. 44.

11 h 26 . Richtet sich plötzlich auf.

11 h 32. Legrt sich auf den Bauch.

11 h 34. Puls 250, Resp. 99.
$11 \mathrm{~h} 38$. Hat sich aufgerichtet.

$11 \mathrm{~h} \mathrm{40.} \mathrm{Legt} \mathrm{sich} \mathrm{auf} \mathrm{den} \mathrm{Bauch.}$

12 h 10 . Richtet sich plötzlich wieder auf. 12 h 12 . Legt sich wieder nieder.

$12 \mathrm{~h}$ 18. Hat sich wieder aufgerichtet. Hüpft umher.

12 h 34 . Legt sich wicder.

12 h 35. Resp. 39.

I h 25. Liegt auf dem Bauche. Resp. 39.

1 h 30 . Richtet sich auf.
11 h 10 . Sitzt ruhir.

11 h 12. Legt sich auf den Bauch.

11 h 14. Starke Peristaltik. Resp. 41.

11 h 18. Durehfall.

11 h 20 . Puls 247.

11 h 50. Erhält 5,4 ccm.
18. 3. 1912. $1800 \mathrm{~g}$.

12 h 00 . Legt sich auf den Bauch.

$12 \mathrm{~h} 20$. Hat sich aufgerichtet.

$12 \mathrm{~h} 30$. Sitzt hockend, ganz benommen. Ohrgefiasse stark erwoitert.

12 h 50. Puls 260, Resp. 37. I Jurchfall.
11 h 00 . Sitzt ruhig.

11 h 15 . Puls 260, Resp. 86

$11 \mathrm{~h} \mathrm{20.} \mathrm{Legt} \mathrm{sich} \mathrm{auf} \mathrm{den} \mathrm{Bauch.}$

11 h 40 . Erhält $5,4 \mathrm{ccm}$.

11 h 50. Resp. 96.

12 h 15. Liegt ganz beoommen auf dem Bauche.

12 h 30 . Puls 260, Resp. 43.
19. 3. 1912. $1800 \mathrm{~g}$.

12 h 42. Sitzt hockend, sehr mïde.

12 h 50 . Leurt sich auf den Bauch.

12 h 55. Hat sich augerichtet. Ohrgefässe stark erweitert.

1 h 00 . Sitzt hockend, sehr müde.

1 h 15 . Legt sich auf den Bauch.

$1 \mathrm{~h} 30$. Wird in den Käfig gesetzt.

20. 3. $1912.1800 \mathrm{~g}$

11 h 10. Hat sich auf den Bauch gelegt. 11 h 15. Puls 245, Resp. 80.

$11 \mathrm{~h} 40$. Erhält $5,4 \mathrm{ccm}$.

12 h 25 . Resp. 80. Hat die ganze /seit ruhig gesessen, schr müde. Vorderpfoten gleiten aus.

12 h 30. Sitzt zusamngekauert.

12 h 40 . Hat sich aufreriehtet.

$1 \mathrm{~h} 00$. Puls 200, Resp. 57.

$1 \mathrm{~h} 08$. Liegt auf dem Bauche.

1 h 15 . Hat sich aufgerichtet.

1 h 20 . Wird in den Käfig gesetzt.

21. 3. $1912.1800 \mathrm{~g}$.

11 h 10 . Sitzt ruhig.

$11 \mathrm{~h}$ 15. Legt sich auf den Bauch.

11 h 20. Puls 220, Resp. 37.

11 h 30 . Erhält $5,4 \mathrm{ccm}$.

12 h 03 . Resp. 32. Hat sich auf den Bauch gelegt.

$12 \mathrm{~h}$ 10. Hat sich aufgerichtet.
12 h 40. Sitzt zusammengekauert, sehr miide.

12 h 50 . Sitzt hockend, sehr benommen.

1 h 05 . Jeegt sich auf den Bauch.

$1 \mathrm{~h} 28$. Hat wälrend der ganzen heit auf dem Bauch gelegen. Resp. $\mathbf{4 2 .}$

$1 \mathrm{~h} \mathrm{30.} \mathrm{Wird} \mathrm{in} \mathrm{den} \mathrm{Kätig} \mathrm{gelegt.}$

22. 3. 1912. $1800 \mathrm{~g}$.

11 h 10. Sitzt aufrecht.

11 h 18. Legt sich auf den Bauch.

11 h 30. Puls 260, Resp. 66. Kussmaul.

11 h 50. Erhält $5,4 \mathrm{ccm}$.

11 h 5j. Hat sich auf den Bauch gelegt. 12 h 00 . Resp. 58, sehr flach.

12 h 30 , Hat die ganze Zeit iiber auf dem Bauche gelegen, sehr benommen.
12 h 40 . Cornealreflex erhalten. Reagirt auf Zupifen der Spürliaare nicht, wohl aber auf Kneifen des Schwanzes, versucht sich aufzurichten, Vorderpfoten gleiten aus. 


\section{3. $1912.1800 \mathrm{~g}$.}

11 h 25. Liegt auf dem Bauche. Beim Berühren, um es aus dem Käligg zu nehmen, heftiger $O_{p}$ is thotonus.

11 h 35. Liegt gany ermattet auf der Seite. $\left.\begin{array}{lll}11 & \text { h } & 50 . \\ 12 & 00 .\end{array}\right\}$ Tonische clonische Krämpfe.

12 h 15 . Liegt auf der Seite, ganz schwach athmend.

12 h 48 . Tetanus. Athmung steht.

12 h 50 . A thmet wieder ganz schwach.

12 h 55 . Furchtbare Dyspnoe.

$1 \mathrm{~h} 00$. Athmung steht. Ierz nicht zu fühlen. Tod nach 7 mal 3 cem.

\section{Methylalkolıol, acetonfrei, š cem pro Kilo.}

31. 5. 1912. Kaninchen, $2300 \mathrm{~g}$.

1 h $05.11,5 \mathrm{ccm}$.

1 h 20. Cnruhig. Hüpft umber.

$1 \mathrm{~h} 30$. Wird in den Kiafig gesetzt.

1. 6. $1912.2200 \mathrm{~g}$.

$12 \mathrm{~h} 10.11 \mathrm{ccm}$.

12 h 15 . Ohrgefässe stark erweitert, legt sich auf den Bauch. Respiration fliegend.
12 h 40. Richtet sich auf und hüpft umher.

12 h 55 . Ieicht benommen.

\section{6. 1912 . $2100 \mathrm{~g}$.}

11 h $15.10,5 \mathrm{ccm}$.

$11 \mathrm{~h} 45$. Hüpft umher.

$11 \mathrm{~h} \mathrm{50.} \mathrm{Sitzt} \mathrm{aufrecht.} \mathrm{Leicht} \mathrm{benommen.}$

11 h 55. Legt sich auf den Bauch. Sehr benommen.
12 h 10 . Hat sich aufgerichtet.

12 h 15 . Hïpft umher.

12 h 25 . Sitzt aufrecht. l,eicht benommen. 12 h 50 . Hüpft umher.

\section{6. 1912. $2000 \mathrm{~g}$.}

$11 \mathrm{~h} \mathrm{40.} \mathrm{Huipft} \mathrm{umher.}$

$11 \mathrm{~h} 50.10 \mathrm{ccm}$.

$12 \mathrm{~h} 00$. Legt sich auf den Bauch. Hintertheil seitlich.

12 h 20 . Richtet sich plötzlich auf.

12 h 30 . Sitzt hockend.

12 h 45 . Sitzt aufrecht.

5. 6. $1912.2000 \mathrm{~g}$.

11 h $40.10 \mathrm{ccm}$.

$12 \mathrm{~h} 02$. Hat sich auf den Bauch gelegt. Ganz benommen.

12 h 15 . Richtet sich plötzlieh auf und hüpft davon.
12 h 40. Sucht Stiitze an der Wand. Fällt um. Hintertheil seitlich. Schaumiges Nasensecret.

\section{6. $1912,2000 \mathrm{~g}$.}

11 h 15. Sitzt aufrecht. I/eicht benommen.

11 h 20. Hat sich auf den Bauch gelegt.

11 h 25. Resp. 48. Kussmaul.

$11 \mathrm{~h} 40.10 \mathrm{cem}$.

$11 \mathrm{~h}$ 50. Hat sich auf den Bauch grelegt.

12 h 00. Richtet sich auf und hüpt. Taunelt.
12 h 10. Vorderpfoten gleiten aus. Ganz benommen. Liegt auf dem Bauche.

12 h 12. Richtet sich wieder auf und büpft davon.

\section{6. $1912.2000 \mathrm{~g}$.}

11 h $25.10 \mathrm{ecm}$.

$11 \mathrm{~h} 40$. Liest ganz benommen auf dem Bauche. Ohrgefässe stark erweitert.

$11 \mathrm{~h} 50$. Hat sich plit:lich aufgerichtet und hüpft urnher.

12 h 15 . Wankt, fällt um, richtet sich aber gleich witeder auf. Sch aumiges Nasensecret.

12 h 25 . Sehr benommen. Leichte Dyspnoe.

12 h 26 . Wankt, fällt um, richtet sich aber gleich wieder auf.
12 h 40. Liegt auf dem Bauche. Hintertheil seitlich gelähmt. Starke Peristaltik.

12 h 50. Nach mehreren vergeblichen Versuchen ist es ihm gelungen,

12 h 55 . Wankt. sich wieder aufzurichten.

1 h 00 . Sitzt ganz benommen.

1 h 05 . Respiration stossweise. Schaumiges Nasensecret. 


\section{6. $1912.1900 \mathrm{~g}$.}

$12 \mathrm{~h}$ 15. Hüpft langsam umher. Starker Haarausfall.

12 h $30.9,5 \mathrm{ccm}$.

$12 \mathrm{~h}$ 35. Wankt, legt sich auf den Bauch.

$1 \mathrm{~h} 00$. Iiegt ganz benommen auf dem Bauche.
1 h 05 . Starke P'eristaltik.

1 h 15. Ilat sich aufgerichtet. Sehr benommen. Wankt, fiallt auf die Seite.

\section{6. $1912.1700 \mathrm{~g}$.}

$11 \mathrm{l}_{\mathrm{l}}$ 45. Hüpft umber. Iseicht benomnen. 11 h $50.8,5 \mathrm{ecm}$.

$11 \mathrm{~h} 55$. Hüpft fort. Benommen. Ohrgefässe stark erweitert.

12 h 30 . Ganz benommen.

$1 \mathrm{~h} \mathrm{10.} \mathrm{Wankt,} \mathrm{fällt} \mathrm{auf} \mathrm{die} \mathrm{Seite.}$
1 h 15. Richtot sich plötzlich auf. Wankt, sucht Stiitze an der Wand. Schaumiges Tasensecret.

$1 \mathrm{~h} 20$. Sitzt zusamengekauert. Kopf gestiitz.t.

$1 \mathrm{~h} 30$. Wird in den Käfig gesetzt.
$11 \mathrm{~h} 50$. Sehr benommen.

11. 6. 1912. $1600 \mathrm{~g}$.

$11 \mathrm{~h} 55.8 \mathrm{ccm}$.

$12 \mathrm{~h} 20$. Sityt ganz benommen.
12 h 40. Fiall um.

$12 \mathrm{~h} 45$. Hat sich wieder aufgerichtet.

12. 6. 1912. Todt im Käfig vorgefunden, $1500 \mathrm{~g}$, nach $10 \mathrm{mal} 5 \mathrm{ccm}$.

\section{Aethylalkohol, 5 ecm pro Kilo.}

31. 5. 1912. Kaninchen, $2000 \mathrm{~g}$.

$11 \mathrm{~h} 30.10 \mathrm{ccm}$.

11 h 40. Resp. 100, fliegend. Stiitzt den hopf.

$12 \mathrm{~h} 00$. Sehr benommen. liegt auf dem Bauche.

$1 \mathrm{~h} 05$. Hat sich aufrerichtet und hüpt davon.

11 h 20. Benommen.

1. 6. $1912.2000 \mathrm{~g}$.

$11 \mathrm{~h} 30.10 \mathrm{ccm}$.

11 h 40. Resp. 63.

$12 \mathrm{~h} 30$. Sitzt ganz benommen. Wankt.

3. 6. $1912.2000 \mathrm{~g}$.

$11 \mathrm{~h} \mathrm{15.} \mathrm{Benommen.} \mathrm{Liegt} \mathrm{anf} \mathrm{dem} \mathrm{Bauche.}$

11 h 20 . Resp. 200, fliegend.

$11 \mathrm{~h} 30.10 \mathrm{ccm}$.

$11 \mathrm{~h} 45$. Liegt gant benommen auf dem

12 h 10 . Desgleichen.

12 h 45 . Descrleichen.

$1 \mathrm{~h} 00$. Hat sich aufgerichtet.

1 h 05 . Sucht Stuitze an der Wand. Bauche.

4. 6. $1912.2000 \mathrm{~g}$.

$11 \mathrm{~h} 40.10 \mathrm{ccm}$.

11 h 50. Sitzt ruhig. Ohrgefässe stark erweitert.

$12 \mathrm{~h} 00$. Jjiegt auf dem Bauche. Hintertheil seitlich golähmt.

12 h 50. Liegt ganz gelähmt.

\section{6. $1912.2000 \mathrm{~g}$.}

$11 \mathrm{~h} 50.10 \mathrm{ccm}$.

$1 \mathrm{~h} 00$. Sitzt hockend.

12 h 15. Liegt auf dem Bauche, ganz be-

1 h 10. Sitzt hockend, ganz benommen. nommen.

$1 \mathrm{~h}$ 15. Wird in den Käfig gesetzt.

6. 6. $1912.1800 \mathrm{~g}$.

11 h 20. Sitzt ruhig. Ohrgefässe stark erweitert.

11 h $35.9 \mathrm{ccm}$.

12 h 40. Resp. 37. Iiegt auf dem Bauche. Hintertheil gelähmt. Versucht sich aufzurichten, fällt aber immer wieder auf die Seite.
1 h 08 . Schläft tief. Resp. 43.

$1 \mathrm{~h}$ 10. Hat sich aufgerichtet.

$1 \mathrm{~h} 15$. Wankt.

$1 \mathrm{~h} 20$. Fällt auf die Seite.

1 h 22 . Richtet sich wieder auf, fällt aber gleich wieder um.

\section{6. $1912.1800 \mathrm{~g}$.}

$11 \mathrm{~h} 40.9 \mathrm{ccm}$.

12 h 10. Hat sich aufgerichtet. 


\section{6. $1912.1900 \mathrm{~g}$.}

$11 \mathrm{~h} 30.9,5 \mathrm{ccm}$

$12 \mathrm{~h}$ 10. Liegt auf dem Bauche, ganz benommen.

12 h 20 . Hat sich aufgerichtet.

12 h 25 . Sitzt zusammengekauert.
1 h 10. Hat sich aufgerichtet.

$1 \mathrm{~h} 20$. Hüpft umher.

$1 \mathrm{~h} 25$. Sitzt benommen. Sucht Stiitze an der Wand.

$1 \mathrm{~h} 30$. Wird in den Käfig gesetzt.

10. 6. $1912.1900 \mathrm{~g}$.

11 h $50.9,5 \mathrm{ccm}$.

$12 \mathrm{~h} 10$. Sitzt zusammengekauert, ganz benommen.
$12 \mathrm{~h} 40$. Sitzt zusammengekauert, ganz benommen.

\section{6. $1912.1900 \mathrm{~g}$.}

$11 \mathrm{~h} 00.9,5 \mathrm{ccm}$.

12 h 12. Fällt auf die Seite.

$11 \mathrm{~h} 30$. liegt gan benommen auf dem Bauche.

12 h 00. Liegt auf dem Bauche. Hintertheil gelähmt.

$12 \mathrm{~h} 10$. Hat sich aufgerichtet.

$12 \mathrm{~h} 20$. Richtet sich wieder auf.

12 h 22 . Fällt auf die Seite, ricitet sich aber bald wieder auf.

12 h 30 . Wankt.

12 h 50. Sitzt hockend. Ganz benommen.

12. 6. $1912.1900 \mathrm{~g}$.

$11 \mathrm{~h} 45.9,5 \mathrm{ccm}$.

$12 \mathrm{~h}$ 00. Liegt auf dem Bauche.

$12 \mathrm{~h} 30$. Lient auf der Seite. Ganz benommen.
12 h 50. Liegt auf der Seite. Hintertheil gelähmt.

$12 \mathrm{~h} 55$. Hat sich aufoerichtet. Ganz benommen. Vorderpfoten gleiten aus

13. 6. $1912.1700 \mathrm{~g}$.

11 h $30.8 .5 \mathrm{ccm}$.

12 h 40. Resp. 46.

$12 \mathrm{~h}$ 00. Liegt gelähmt auf dem Bauche.

$12 \mathrm{~h} \mathrm{l}$ 15. Liegt gelähmt auf der Seite.

14. 6. 1912. Todt im Käfig vorgefunden, nach $12 \mathrm{mal} 5 \mathrm{cc}$ cm.

\section{Methylalkohol, acetonfrei, 6 ecm pro Kilo.}

19. 6. 1912. Kaninchen $1600 \mathrm{~g}$.

12 h $05.9,6 \mathrm{ccm}$.

12 h 20 . Hat sich gelegt. Ilustenstösse. Kopf aurecht.

12 h 30. Liegt ganz ruhig, benommen. Scluamiges Nasensecret.

$12 \mathrm{~h} \mathrm{40.} \mathrm{Beim} \mathrm{Versuch} \mathrm{sich} \mathrm{aufzurichten}$ gleiten die Vorderpfoten aus. Resp. 50.

$12 \mathrm{~h} 45$. Rirhtet sich auf, fällt aber gleich wieder um.
$12 \mathrm{~h} \mathrm{47.} \mathrm{Hiegt} \mathrm{auf} \mathrm{dem} \mathrm{Bauche.} \mathrm{Hinter-}$ theil seitlich.

12 h 50 . Hat sich aufgerichtet.

1 h 00 . Sitzt ruhig, leicht benommen.

1 h 10. Sitzt ruhig, leicht benommen. Resp. 41 .

1 h 12 . Richtet sich plötzlich vorne auf. 1 h 15. Sitzt ruhig.

$1 \mathrm{~h} 30$. Wird in den Kifig gesetzt.
20. 6. $1912,1500 \mathrm{~g}$.

$12 \mathrm{~h} 30.9 \mathrm{ccm}$.

21. 6. $1912.1400 \mathrm{~g}$.

11 h $55.8,4 \mathrm{ccm}$.

12 h 20. Liegt auf dem Bauche.

12 h 40. Grosse Unruhe. Versucht sich aufzurichten, aber vergeblich.

\section{6. 1912, $1400 \mathrm{~g}$.}

11 h 15 . Jiegt auf dem Bauche. Hintertheil seitlich.

11 h $40.8,4 \mathrm{ccm}$.

12 h 20 . Liegt auf dem Bauche.

$12 \mathrm{~h} 50$. liegt auf der Seite. Ganz benommen. Resp. $3 i$.

1 h. 25. Bronchialrasseln. Dyspnoe. $1 \mathrm{~h} 30$. Wird in den Käfig gelegt.

24. 6. $1912,1300 \mathrm{~g}$.

$11 \mathrm{~h}$ 20. Liegt auf dem Bauche. 'Ganz be- $11 \mathrm{~h} 45.7,8 \mathrm{cem}$. nommen. Schaumiges Nasen- 11 h 55. liegt auf der Seite, gelähmt. secret. Leichte Iyspnoe.

11 h 30 . Resp. 24 .

11 h 36 . Richtet sich auf.
$1 \mathrm{~h} 20$. Desgleichen.

$1 \mathrm{~h} 25$. Wird in den Käfig gelegt.

25. 6. 1912. Todt vorgefunden, nach 5 mal 6 ccm. 


\section{Aethylalkohol, 6 ccm pro Kilo.}

$12 \mathrm{~h} 15.11,4 \mathrm{ccm}$.

19. 6. 1912. Kaninchen, $1900 \mathrm{~g}$.
$12 \mathrm{~h}$ 30. Liegt auf dem Bauche. Ohrgefïsse stark erweitert.

12 h 40 . Resp. 240

$12 \mathrm{~h} 50$. Lient gelähmt auf der Seite. In den Schwanz gekniffen, springt es auf, fällt aber sofort wieder um.
12 h 55 . Resp. 130.

1 h 00. Resp. 158. Liegt auf der Seite.

$1 \mathrm{~h} 05$. Versucht vergeblich sich aufzurichten.

1 h 15 . Resp. 133. Liegt auf der Seite. I h 30 . Wird in den Käfigr gelegt.
12 h $00.9 \mathrm{ccm}$.

$12 \mathrm{~h} 20$. Sitzt ganz benommen.

12 h 30 . Hat sich anfgerichtet, wankt und fiillt um.

20. 6. $1912.1700 \mathrm{~g}$

$12 \mathrm{~h} 45.10,2 \mathrm{ccm}$.

21. 6. 1912 . $1500 \mathrm{~g}$.

\section{6. $1912.1400 \mathrm{~g}$.}

11 h 15. Sitzt hockend.

11 h $50.8,4 \mathrm{ccm}$.

12 h 10. Hat sich auf den Bauch gelegt.

12 h 25 . Sitzt hockend.

$12 \mathrm{~h} 35$. Taumelt.

$12 \mathrm{~h} \mathrm{40.} \mathrm{Fällt} \mathrm{um.}$

12 h 45 . Richtet sich auf.
12 l. 35. Versucht fortzuhüpfen, fällt aber immer wieder um.

12 h 40. Jiegt auf dem Bauche.

$1 \mathrm{~h} 00$. Sucht Stiitze an der Wand, legt sich auf den Bauch, Hintertheil seitlich.

1 h 20. Richtet sich plitzlich auf, fillt aber rrleich wieder um.

1 h 30 . Wird in den Kitifg gelert.

\section{6. $1912.1350 \mathrm{~g}$.}

11 h 25. Sitat ruhig. Resp. 53.

$11 \mathrm{~h} 32$. Hat sich gelegt. Hintertheil seitlich.

11 h $50.8,1 \mathrm{ccm}$.

12 h 00. Hat sich auf den Bauch gelegt.

12 h 20. Sitzt ruhig, benommen.

$12 \mathrm{~h} 30$. Liegt auf der Seite.

12 h 40. Resp. 120.
$12 \mathrm{~h} 50$. Hat sich aufgerichtet. Hüpft davon. Wankt, taumelt und faillt um.

1 h 05 . Riclitet sich auf, versuch tortzuhüpfen, die Vorderpfoten grleiten aus, fällt auf die Seite.

$1 \mathrm{~h} 20$. Wird in den hitig gesetot.
11 h 45 . Erhält $7,8 \mathrm{ccm}$.

12 h 00 . Yicgt auf dem Bauche. Hintertheil scitlich.

12 h 20 . Versucht sich aufzurichten. Vorderpfoten gleiten aus, lerit sich wicder auf den Bauch.
25. 6. $1912.1300 \mathrm{~g}$.

12 h 45 . I,iegrt auf dem Bauche. Jintertheil gelähmt.

I h 20. Springt auf, fält um.

$1 \mathrm{~h} 30$. Versucht veroblich sich aufzurichten, dic Beine grleiten aus. Wird in den kifig gresetzt.

26. 6. 1912.1300 in.

12 h 10 . Erhält $7,8 \mathrm{~cm}$.

$12 \mathrm{~h} 30$. Sitzt hockend, ganz benommen. Ohrrefässe stark erweitert.

12 h 35 . Liegrt ganz benommen auf dem Bauche, Hintertheil seitlich gelähmt. Resp. 90.

$12 \mathrm{~h} \mathrm{50.} \mathrm{Liegt} \mathrm{völlig} \mathrm{gelähmt} \mathrm{auf} \mathrm{der} \mathrm{Seite.}$
1 h 00 . Versucht verceblich sich aufzurichten und fortzuhüpfen, fällt inmer wieder um.

1 h 20 . Jiegt gelähnt auf ter Seite.

1 h 25 . Selir unruhig. Versurht fortzuhiipfen, fallt immer wieder um.

1 h 30. In den Kaifir gelegrt.

27. 6. $1912.1300 \mathrm{~g}$.

$11 \mathrm{~h} 05$. Erhält 7,8 $\mathrm{cm}$.

11 h 30 . Sitzt ruhig hockend.

$11 \mathrm{~h} 40$. Liegt auf dem Bauche. Hintertheil seitlich gelähmt.
$11 \mathrm{~h} 50$. Vollstiandig gelïhmt.

12 h 50 . Vollstiandig gelihmt.

12 h 5ó. Kirhtet sich auf, fäll aber sofort u.m.

28. 6. $1912.1250 \mathrm{~g}$.

11 h 25. Legt sich auf den Bauch, streckt die Ilinterbeine weit aus.

$11 \mathrm{~h} 35$. Erhält $7,5 \mathrm{ccm}$.

$12 \mathrm{~h}$ (00. Liegt auf dem Bauche. Versucht sich aufzurichten. Wankt.

12 h 05. liegt auf dem Bauche, Hinterbeine weit ausgrestreckt.
$12 \mathrm{~h} \mathrm{20.} \mathrm{Jjegt} \mathrm{auf} \mathrm{der} \mathrm{Seite} \mathrm{vollständig}$ gelahnt.

12 h 30 . Resp. 37.

12 h 50. Hat sich aufrerichtot.

12 h 55. Liegt auf dem Baurhe.

$1 \mathrm{~h} 00$. Wird in den käfig gelegt.

29. 6. 1912. Todt rorgefunden. 9 mal f ecnt pro Kilo. 


\section{Yethylalkohol, 8 eem pro Kilo.}

\section{6. 1912 . Kaninchen, $1600 \mathrm{~g}$.}

11 h 20 . Erhält $12,8 \mathrm{ccm}$.

11 h 50. Liegt auf dem Bauche. Resp. 240.

$11 h_{1} 55$. Richtet sich auf.

12 h 05 . Sitzt hockend.

12 h 10. Richtet sich auf. Hüpft davon.
12 h 20. Iüpft umher. Reflexerregbarkeit erhöht.

12 h 40 . Sitzt ruhig, leicht benommen.

12 h 45 . Huipft umher.

$12 \mathrm{~h} 50$. Uüpft umher.

12 h 55 . Sitat leicht benommen.

28. 6. 1912 . $1600 \mathrm{~g}$.

11 h 45 . Erbält $12,8 \mathrm{ccr}$.

11 h 55. Liegt auf dem Bauche. Hintertheil seitlich.

12 h 00 . Hat sich aufgeriehtet, hüpft davon.

12 h 05 . Iert sich auf den Bauch, richtet sich aber bald wieder auf, hüpft davon. Grosse Linruhe.
$12 \mathrm{~h} 10$. Hüpt fortwährend umher, sehr erregt.

$12 \mathrm{~h} 30$. Hüpft fortwährend umher, fällt häufig um, richtet sich aber sofort wieder auf.

$1 \mathrm{~h} 00$. Wird in den Käfig gesetzt.

\section{6. $1912.1600 \mathrm{~g}$.}

$11 \mathrm{~h} 40$. Liegt auf dem Bauche, leicht benommen.

11 h 45 . Resp. 47.

$11 \mathrm{~h} 52$. Richtet sich auf.

11 h 53. Wankt, leut sich auf den Bauch.

12 h 20 . Erbält $12,8 \mathrm{ccm}$.
12 h 30 . liegt golähmt auf den Bauche.

12 h 35 . Nystagmus. Tersucht vergeblich sich aufzurichten.

12 h 52 . Hat die ganze Zeit über gelühmt auf der Seite gelegen. Resp. 101.

$1 \mathrm{~h} 20$. Wird in den Käfig gelegt.

1. 7. 1912. 'Todt vorgefunden. 3 mal 8 cem pro Kilo.

\section{dethylalkohol, 8 eem pro Kilo.}

\section{6. 1912. Kaninchen, $1600 \mathrm{~g}$.}

11 h 15 . Firhält $12,8 \mathrm{ccm}$.

$11 \mathrm{~h} 30$. Liegrt auf dem Bauche, fliegende A thmung. Resp. 300.

II 5 5. Liegt gelïhnt auf dem Bauche. Resp. 250.
$12 \mathrm{~h} 05$. Liegt gelïhmt auf der Seite. Resp. 250 .

12 h 35 . Versucht vergeblich sich aufzurichten.

12 h 40. I,iegt völligr gelïhmt auf der Seite.

25. 6. $1912.1500 \mathrm{~g}$.

$11 \mathrm{~h} 50$. Erhält $12 \mathrm{~cm}$.

12 h 00 . Liegt auf dem Bauche, fliegende Athmung.

12 h 15. Iiegt auf den Bauche ganz benommen.

29. 6. $1912.1500 \mathrm{~g}$.

$11 \mathrm{~h} \mathrm{40.} \mathrm{Liegt} \mathrm{auf} \mathrm{dem} \mathrm{Bauche,} \mathrm{fliegende}$ Athmung.

$11 \mathrm{~h} 48$. Hat sich aufgerichtet.

12 h 25 . Erhält $12 \mathrm{ccm}$.
12 h 55 . Hat wïhrend der ganzen zeit ruhig aur dem Bauche gelegen.

1 h 00 . Wird in den Käfig gelegt.

\section{7. 1912. Todt vorgefunden. 3 mal 8 ecm pro Kilo.}

\section{Aethylalkohol, 10 cem pro Kilo.}

\section{7. 1912 . Kaninchen, $1600 \mathrm{~g}$.}

11 h 25. Frhält $16 \mathrm{cem}$.

$11 \mathrm{~h} 55$. Liegrt völlig gelähmt auf der Seite.

$11 \mathrm{~h} 35$. Liegt ganz benommen auf dem $12 \mathrm{~h} 15$. Resp. 41. Liegt völlig gelälınt auf Bauche. der Seite.

11 h 45. Resp. 73. Liegt auf dem Bauche. 1 h 20. Wird in den Fäfig gelegt.

3. 7. 1912. Am Morgen todt vorgefunden.

\section{Methylalkohol, acetonfrei, 10 een pro Kilo.}

2. 7. 1912. Kaninchen, $1500 \mathrm{~g}$.

$11 \mathrm{~h} 15$. Frrhält 15) ccm.

11 h 20. Leert sich auf den Bauch.

$11 \mathrm{~h} 35$. Liegt gelühmt auf der Seite. Kuck kungen der Kalsmuskulatur. Nystagmus.
11 h 40. Resp. 130, dyspnoisch.

11 h 55. Reflexerregbarkeit gesteigert.

12 h 05. Resp. 150. Tracheairassein. 


\section{7. 1912 (Fortsetzung).}

12 h 10. Grosse Unrube, versucht vergeblich sich aufzurichten.

$12 \mathrm{~h} 25$. Versucht sich aufzurichten, fällt aber stets wieder auf die Seite.

12 h 45. Liegt auf der Seite.

3. 7. $1912.1400 \mathrm{~g}$.

12 h 00 . Sitzt rubig, leicht benommen.

12 h 15 . Resp. 77.

12 h 20 . Hüpft davon.
1 h 05 . Liegt auf der Seite. Zittern und Zuckungen.

1 h 20 . Desgleichen.

$1 \mathrm{~h} 30$. Wird in den Käfig gesetzt.

\section{$1 \mathrm{~h} 15$. Hat während der ganzen Zeit theils ruhig gesessen und ist theils uin- hergehüpft. \\ $1 \mathrm{~h} \mathrm{20.} \mathrm{Wird} \mathrm{in} \mathrm{den} \mathrm{Käfig} \mathrm{gesetzt.}$}

4. 7. $1912.1200 \mathrm{~g}$.

12 h 40. Hüpft umber.

5. 7. $1912.1200 \mathrm{~g}$.

12 h 00 . Ganz mobil. Hüpft umher.

6. 7. $1912.1300 \mathrm{~g}$.

12 b 00 . Ganz mobil, hüpft umber.

$12 \mathrm{~h} \mathrm{10.} \mathrm{Hüpft} \mathrm{umher.}$

$12 \mathrm{~h} 30$. Sitzt ruhig.

$11 \mathrm{~h} 05$. Ganz mobil.
12 h 45 . Hüpft umher.

$1 \mathrm{~h} 00$. Wird in den Käfig gesetzt.

S. 7. $1912.1300 \mathrm{~g}$.

i 12 h 05 . Hüpft umher.

9. 7. $1912.1950 \mathrm{~g}$.

12 h 00 . Ganz mobil. Sitzt ruhig. Das Thier ist nicht eingegangen.

\section{Aethylalkohol, 10 ccm pro Kilo.}

5. 7. 1912. Kaninclien $1400 \mathrm{~g}$.

$12 \mathrm{~h} 55$. Frhält $14 \mathrm{ccm}$.
$1 \mathrm{~b}$ 05. Liegtganz gelähmt auf demBauche.

6. 7. $1912.1250 \mathrm{~g}$. Todt vorgefunden.

Hethylalkohol, aretonfrei, 10 cem pro Kilo.

5. 7. 1912. Kaninchen $1500 \mathrm{~g}$.

12 h 50 . Firhält $15 \mathrm{ccm}$.

$1 \mathrm{~h} 00$. Hat sich gelegt.

1 h 05. Richtet sich auf, hüpft davon.

$1 \mathrm{~h} \mathrm{10.} \mathrm{Grosse} \mathrm{Unruhe,} \mathrm{Reflexerreg-}$

barkeit gesteigert.

11 h 30 . Sitzt rubig.

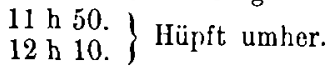

\section{7. $1912.1500 \mathrm{~g}$.}

12 h 13 . Sitzt ruhig, leicht benommen. 12 h 45 . Hiipft ganz munter umher.

8. 7. $1912.1300 \mathrm{~g}$.

$11 \mathrm{~h} 05$. Sitzt ruhig, leicht benommen. | $11 \mathrm{~h} 15$. Hüpft munter umher.

9. 7. $1912.1250 \mathrm{~g}$.

11 h 20. Ganz munter, hïpft umher. $12 \mathrm{~h} 20$. Hüpft umber.

11 h 50. Leicht benommen, hat sich gelegt. $12 \mathrm{~h} 30$. Hat sich gelegt.

10. 7. $1912,1300 \mathrm{~g}$.

$11 \mathrm{~h} 30$. Ieicht benommen. $12 \mathrm{~h} 20$. Richtet sich auf, wird in den

12 h 15. Liegt auf dem Bauche. Reflexerregbarkeit erhöht. Käfig gesetzt.

11. 7. $1912.1300 \mathrm{~g}$.

$12 \mathrm{~h}$ 15. Sitzt aufrecht, leicht benommen. ! $1 \mathrm{~h} 30$. Wird in don häfig gresetzt.

15. 7. $1912,1300 \mathrm{~g}$.

11 h 30 . Ganz munter, hïpft umher.

$1 \mathrm{~h} \mathrm{30.} \mathrm{Wird} \mathrm{in} \mathrm{den} \mathrm{Kiffig} \mathrm{nesetzt.}$

Das Thier ist nicht eingegangen. 
Aethylalkohol, 10 cem pro Kilo.

$1 \mathrm{~h}$ 1.5. Frhält $13 \mathrm{ccm}$.

8. 7. 1912 . Kaninchen $1300 \mathrm{~g}$.

9. 7. $1912.1200 \mathrm{~g} . \quad$ Todt vorgefunden.

Methylalkohol, acetonfrei, 10 cem pro Kilo.

S. 7. 1912. Kaninehen $1400 \mathrm{gr}$.

$1 \mathrm{~h} 10$. Erhält $14 \mathrm{ccm}$.

9. 7. $1912.1300 \mathrm{~g}$.

$11 \mathrm{~h}-1 \mathrm{~h} 00$. Hüpft fortwäbrend umher.

10. 7. $1912,1300 \mathrm{~g}$.

11. h $30-12$ h 15. Sehr unruhig, hiipft fortwährend umher.

15. 7. $1912.1400 \mathrm{~g}$.

Iüpft fortwährend umher.

$11 \mathrm{~h} 30$. Hüpft umher.

16. 7. $1912.1400 \mathrm{~g}$.

$12 \mathrm{~h} 00$. sitzt ruhig, aufrecht. 12 h 30 . Hüpft umher.

17. 7. $1912 . \quad 1350 \mathrm{~g}$.

Ohrgefässe stark crweitert, hüpft umher. Das Thicr ist nicht eingregangen.

Aethylalkohol, 10 cem pro Kilo.

10. 7. 1912. Kaninchen $1300 \mathrm{~g}$.

12 h 50 . Lirhält $13 \mathrm{ccm}$.

$12 \mathrm{~h} 55$. Liegt auf dem Bauche, granz be-

I h 15. Liegt vüllig geliahmt auf der noinmen. Resp. 240.

Seite.

11. 7. 1912. Todt vorgefunden.

Metlyylalkohol, acetonfrei, $10 \mathrm{cem}$ pro Kilo.

10. 7. 1912. Kaninchen 1400 ․

$12 \mathrm{~h} 55$. Erhält $14 \mathrm{ccm}$.

1 h 20. Liegt auf dem Bauche.

$1 \mathrm{~h} 05$. Sitzt huckend. Resp. fliegend. $1 \mathrm{~h} 30$. Wird in den Kiffig grelort.

11. 7. $1912.1300 \mathrm{~g}$.

11 h 05 . Liegt benommen auf dem Bauche.

11 h 30 . Hat sich aufgerichtet, hiipft davon.

12 h 30 . Luegt sieh auf den Bauch.

1 h 00 . Richtet sich auf. Sitzt ruhigr.

$1 \mathrm{~h} 30$. Wird in den Käfig gesetzt.

12. 7. $1912.1200 \mathrm{~g}$.

$11 \mathrm{~h} 30--11 \mathrm{~h} 45$. Hüpft munter umher.

13. 7. $1912.1100 \mathrm{~g}$.

11 h 30. Hat sich grelegt.

11 h 50 . Richtet sich auf und hüpft umher.

$12 \mathrm{~h} 00$. Wird in den Käfig gesetzt.

16. 7. $1912.1100 \mathrm{~g}$.

Hijpft ganz munter umher. Das Thier ist nicht eingegangen.

\section{Aethylalkohol, 10 cen pro Kilo.}

15. 7. 1912. Kaninchen $900 \mathrm{~g}$.

12 h 25 . Erhält $9 \mathrm{ccm}$.

12 h 35. Sitzt ruhig. Kopf srestützt.

$12 \mathrm{~h} \mathrm{45}$. Licgt ganz benommen auf dem

Bauehe. Springt beim lineifen

des Schwanzes auf, fällt aber sofort wieder auf die Seite.

16. 7. 1912. Todt vorgefunden.

\section{Yethylalkolıol, acetonfrei, 10 c'm! pro Kilo.}

15. 7. 1912 . Kaninchen $1000 \mathrm{~g}$.

$12 \mathrm{~h} 00$. Frhält $10 \mathrm{ccm}$.

12 h 45 . Jiegt völlig gelähmt auf der Seite. Reagirt auf kneifen des Schwan- zes, versucht vergeblich, sich aufaurichten.

1 h 10. Ijiegt auf der Seite. Resp. 40. 
$11 \mathrm{~h} 15$. Leicht benommen.

$11 \mathrm{~h} 30$. Hat sich gelegt.

12 h 00 . Richtet sich auf.

$12 \mathrm{~h} 15$. Hüpft umher.

11 h 15. Sitzt aufrecht.

$11 \mathrm{~h}$ 40. Hüpft umber.

$12 \mathrm{~h} 00$. Sitzt ruhig, benommen.

16. 7. $1912.900 \mathrm{~g}$.

12 h 30 . Sitzt aufrecht.

$1 \mathrm{~h} 00$. Hüpft umher.

$1 \mathrm{~h} 20$. Wird in den Käfig gesetzt.

17. 7. $1912.800 \mathrm{~g}$.

$12 \mathrm{~h} \mathrm{50.} \mathrm{Legt} \mathrm{sich} \mathrm{auf} \mathrm{den} \mathrm{Bauch.}$

$1 \mathrm{~h} 00$. Hüpft umher.

$1 \mathrm{~h}$ 15. Wird in den Käfig gesetzt.

18. 7. $1912.800 \mathrm{~g}$.

Ganz mobil, hüpft umher. Das Thier ist nicht eingegangen.

\section{Aethylalkohol, $10 \mathrm{ecm}$ pro Kilo.}

12 h 25 . Erhält $16 \mathrm{ccm}$.

4. 7. 1912. Kaninchen $1600 \mathrm{~g}$.

$12 \mathrm{~h} \mathrm{40.} \mathrm{Sitzt} \mathrm{ruhig,} \mathrm{ganz} \mathrm{benommen.}$ $12 \mathrm{~h} \mathrm{45.} \mathrm{Liegt} \mathrm{gelähmt} \mathrm{auf} \mathrm{der} \mathrm{Seite.}$

5. 7. $1912.1500 \mathrm{~g}$. Todt vorgefunden.

\section{Methylalkohol, acetonfrei, $10 \mathrm{ecm}$ pro Kilo.}

4. 7. 1912. Kaninchen $1500 \mathrm{~g}$.

$12 \mathrm{~h} 30$. Erhält $15 \mathrm{ccm}$.

12 h 40 . Sitat benommen.
12 h 50. Sitzt ruhig, benommen. Reflexerregbarkeit gesteigert.

\section{7. $1912.1400 \mathrm{~g}$. Todt vorgefunden.}

Mischung aus gleichen Theilen.

Mischumg aus gleichen Theilen Methyl- und Aethylalkohol, $10 \mathrm{ccm}$ pro Kilo.

18. 7. 1912. Kaninchen, $850 \mathrm{~g}$.

12 h 50 . Erhält $8.5 \mathrm{ccm}$.

1 h 00. Liegt auf dem Bauche. Resp. fliegend.

$1 \mathrm{~h} \mathrm{15}$. Liegt gelähmt auf der Seite.

19. 7. $1912.700 \mathrm{~g}$.

$11 \mathrm{~h} 30$. Sitat ruhig, leicht benommen.

11 h 40 . Hüpft davon.

$11 \mathrm{~h} \mathrm{50.} \mathrm{Sitzt} \mathrm{ruhig} \mathrm{aufrecht.}$

$11 \mathrm{~h} \mathrm{40.} \mathrm{Sitzt} \mathrm{aufrecht.}$

$12 \mathrm{~h} \mathrm{00}$. Hat sich auf den Bauch gelegt.
$12 \mathrm{~h} 15$. Sitzt ruhig, ganz benommen. 12 h 20. Sucht Stuitze an der Wand.

20. 7. 1912. $650 \mathrm{~g}$.

12 h 10. Hüpft umher.

22. 7. $1912.600 \mathrm{~g}$.

23. 7. 1912. $750 \mathrm{~g}$.

$11 \mathrm{~h} \mathrm{45.} \mathrm{Ganz} \mathrm{mobil.}$
$11 \mathrm{~h}$ 50. Legt sich plötzlich auf den Bauch. 12 h 05 . Richtet sich auf und hüpft davon.

11 h 30 . Hüpft munter umher.

$$
\text { 25. 7. 1912. } 600 \mathrm{~g}:
$$

$12 \mathrm{~h} 15$. Richtet sich auf und hüpft umher.

26. 7. 1912 . $600 \mathrm{~g}$.

10 h 40 . Sitzt hockend.

$$
\text { | } 10 \mathrm{~h} 52 \text {. Hüpft umher. }
$$

Das Thier ist nicht eingegangen.

\section{Methylalkohol, acetonfrei, $12 \mathrm{ccm}$ pro Kilo.}

17. 7. 12. Kaninchen, $900 \mathrm{~g}$

12 h 20 . Erhält 10,8 ccm.

$12 \mathrm{~h} 30$. Sitzt ruhig, leicht benommen.

12 h 40 . Hüpft umher.

12 h 42. Wankt, fällt um.

12 h 45. Liegt auf dem Bauche.

12 h 50. Richtet sich auf, hüpft davon, wankt, fällt um, richtet sich aber gleich wieder auf.

\section{$12 \mathrm{~h} 55$. Hüpft davon.}

$1 \mathrm{~h} 00$. Liegt auf dem Bauche.

$1 \mathrm{~h} 10$. Versucht vergeblich sich aufzurichten. Hintertheil gelähmt.

$1 \mathrm{~h} 30$. Wird in den Käfig gelegt. 
18. 7. 1912. $900 \mathrm{~g}$.

$12 \mathrm{~h}$ 00. Leicht benommen. Sitzt ruhig. $12 \mathrm{~h} 50$. Sitzt ruhig, leicht benommen.

12 h 40 . Hüpft davon.

12 h 55 . Hitpft ganz munter umher.

19. 7. 1912. $900 \mathrm{~g}$.

$11 \mathrm{~h} 25$. Hüpft ganz munter umher. | $12 \mathrm{~h} 00$. Sitzt ruhig, leicht benommen.

20. 7. $1912.800 \mathrm{~g}$.

11 h 50 . Sitz ruhig.

112 h 15 . Hüpft umher.

11 h 40. Sitzt ruhig, aufrecht.

$11 \mathrm{~h}$ 47. Legt sich auf den Bauch.

$22,7.1912$.

11 h 40. Ganz mobil.

23. 7. 1912. $1000 \mathrm{~g}$.

12 h 30 . Hat sich aufgerichtet. Sitzt leicht benommen.

11 h 45 . Hüpft umher.

12 h 10 . Sitzt ruhig.

1 h 05 . Hüpft umher.

24. 7. 1912, $900 \mathrm{~g}$.

11 h 45. Sitzt ruhig.

Das Thier ist nicht eingegangen.

\section{Methylalkohol Ursache der Vergiftung, 12 ccm pro Kilo.}

24. 7. 1912. Kaninchen, $900 \mathrm{~g}$.

10 h 45 . Erhält $10,8 \mathrm{ccm}$.

$11 \mathrm{~h}$ 20. Liegt gelähmt auf dem Bauche. 11 h 30 . Zuckungen.
$11 \mathrm{~h} 35$. Versucht vergeblich sich aufzurichten.

12 h 40 . Völlig gelähmt auf dem Bauche.

25. 7. 1912. Todt aufgefunden. $800 \mathrm{~g}$.

Methylalkohol, acetonfrei, $14 \mathrm{ccm}$ pro Kilo.

22. 7. 1912. Kaninchen, $900 \mathrm{~g}$.

$1 \mathrm{~h} \mathrm{30.} \mathrm{Erhält} 12,6 \mathrm{~cm}$.

23. 7. $1912.950 \mathrm{~g}$. Liegt völlig gelähmt auf der Seite.

11 h 25 . Versucht vergeblich sich aufzu- 11 h 55. Liegt völlig gelähmt auf dem richten, schiebt" sich nur auf dem Bauche vorwärts. Bauche.

12 h 40 . Liegt völlig gelähmt auf der Seite.

24. 7. 1912. $750 \mathrm{~g}$. Liegt völlig gelähmt auf der Seite.

25. 7. 1912. $750 \mathrm{~g}$. Völlig gelähmt.

26. 7. 1912. Todt vorgefunden.

Wie die vorstehenden Protokolle zeigen, ist der Tod eingetreten bei $3 \mathrm{ccm}$ pro Kilo beim Methylalkohol, acetonfrei, nach 19 Gaben, beim Aethylalkohol nach 23 Gaben, beim Methylalkohol Ursache der Vergiftung nach 7 Gaben; bei $5 \mathrm{ccm}$ pro Kilo beim Methylalkohol, acetonfrei, nach 10 Gaben, beim Aethylalkohol nach 12 Gaben; bei $6 \mathrm{ccm}$ beim Methylalkohol nach 5 Gaben, beim Aethylalkohol nach 9 Gaben; bei $8 \mathrm{cem}$ pro Kilo beim Methyl- und Aethylalkohol gleichzeitig nach 3 Gaben. Bei $10 \mathrm{ccm}$ wurden alle 6 Aethylthiere am nächsten Tage nach der Eingabe todt vorgefunden, während von den Methylthicren nur ein einziges eingegangen ist. Auch $12 \mathrm{ccm}$ Methylalkohol, acetonfrei, wirkten nicht tödtlich. Erst $14 \mathrm{ccm}$ pro Kilo wirkten tödtlich am 4. Tage nach Eingabe.

Bemerkenswerth ist, dass auch das Thier, welches $10 \mathrm{ccm}$ einer Mischung aus gleichen Theilen Aethyl- und Methylalkohol erhalten hat, nicht eingegangen ist, ferner die gesteigerte Peristaltik und 
36 Alexander Langgaard, Die Giftigkeit des Methyl- und Aetbylalkohols.

erhöhte Reflexerregbarkeit, dass aber Krämpfe wie nach dem beschlagnahmten, nach dem acetonfreien Methylalkohol nicht beobachtet wurden.

Das Resultat der Versuche ist kurz folgendes:

In kleinen, täglich wiederholten Dosen ist der Methylalkohol giftiger als der Aethylalkohol, in einmaligen grossen Dosen ist der Aethylalkohol bedeutend giftiger als der Methylalkohol.

In kleinen wiederholten Dosen gegeben, äussert der Methylalkohol eine cumulative Wirkung, die wohl dadurch zu Stande kommt, dass, wie dies bereits von anderer Seite (Harnack) ausgesprochen ist, er von gewissen Theilen des Centralnervensystems angezogen und dort langsam zu Ameisensäure oxydirt wird. 